\title{
Constrained welfare egalitarianism in surplus-sharing problems
}

by

Pedro Calleja, Francesc Llerena and Peter Sudhölter

Discussion Papers on Business and Economics

No. $1 / 2020$

FURTHER INFORMATION

Department of Business and Economics

Faculty of Business and Social Sciences University of Southern Denmark Campusvej 55, DK-5230 Odense M 


\title{
Constrained welfare egalitarianism in surplus-sharing problems*
}

\author{
Pedro Calleja ${ }^{\dagger}$ Francesc Llerena $\ddagger$ and Peter Sudhölter ${ }^{\S}$
}

\begin{abstract}
We introduce the constrained egalitarian surplus-sharing rule $f^{C E}$, which distributes an amount of a divisible resource so that the poorer agents' resulting payoffs become equal but not larger than any remaining agent's status quo payoff. We show that $f^{C E}$ is characterized by Pareto optimality, nonnegativity, path independence, and less first, a new property requiring that an agent does not gain if her status quo payoff exceeds that of another agent by the surplus. We provide two additional characterizations weakening less first and employing consistency, a classical invariance property with respect to changes of population. We investigate the effects of egalitarian principles in the setting of transferable utility (TU) games. A singlevalued solution for $\mathrm{TU}$ games is said to support constrained welfare egalitarianism if it distributes any increment of the worth of the grand coalition according to $f^{C E}$. We show that the set of Pareto optimal single-valued solutions that support $f^{C E}$ is characterized by means of aggregate monotonicity and bounded pairwise fairness, resembling less first.
\end{abstract}

Keywords: Surplus-sharing problem, egalitarianism, Lorenz domination, TU game JEL Classification: C71

\section{Introduction}

The notion of equity has a significant position in surplus-sharing problems, where a quantity of a divisible resource (e.g., money) is divided among a set of agents that believe in egalitarianism as a social value. In this setting, authors have mainly paid attention to resource egalitarianism instead of welfare egalitarianism. The first principle is reached

\footnotetext{
${ }^{*}$ The first two authors acknowledge support from research grants ECO2016-75410$\mathrm{P}(\mathrm{AEI} / \mathrm{FEDER}, \mathrm{UE})$ and ECO2017-86481-P(AEI/FEDER,UE), and the third author acknowledges support from the research grant ECO2015-66803-P (MINECO/FEDER).

${ }^{\dagger}$ Departament de Matemàtica Econòmica, Financera i Actuarial, BEAT, Universitat de Barcelona, e-mail: calleja@ub.edu

${ }^{\ddagger}$ Departament de Gestió d’Empreses, CREIP, Universitat Rovira i Virgili at Reus, e-mail: francesc.llerena@urv.cat

$\S^{\S}$ Department of Business and Economics, University of Southern Denmark, e-mail: psu@sam.sdu.dk
} 
by distributing the available total resource equally among the agents, whereas the latter prioritizes to equalize the welfare of the agents after the allocation process. ${ }^{1}$ Nevertheless, if the amount of resource that has to be distributed is small, it may happen that a rich agent has to transfer some of her money to poorer agents in order to reach welfare egalitarianism. To make our approach to egalitarianism compatible with individual selfinterest, we introduce the constrained egalitarian surplus-sharing rule, $f^{C E}$. Imagine a situation where there is a resource to be divided among a set of agents that are ranked with respect to (w.r.t.) a reference point, representing some objective and measurable feature (sometimes called status quo or welfare). First, agents with the lowest ranking receive everything until they become equal to the second lowest ranked agents, and so forth until the resource is exhausted. Distributing according to $f^{C E}$ can be seen as a way of obtaining end-state fairness. ${ }^{2}$ Many real-life allocation methods promote this positive discrimination towards agents with less status quo. For instance, in the distribution of grants or subsidies by public institutions, families with lower incomes often receive larger scholarships and, subsequent to a natural catastrophe, it is often decided that the more individuals suffer, the more financial support they get.

We first show that $f^{C E}$ can be characterized by four properties: Pareto optimality, meaning that the resource must be exhausted, nonnegativity, imposing awards to be nonnegative, path independence (Moulin, 1987), requiring that the assigned payoffs remain unchanged when applying the rule consecutively to any partition of the resource, and less first, a new property capturing how differently non-identical agents (w.r.t. the status quo) should be treated. This property requires that if the relative welfare difference at the status quo between two agents exceeds the total amount to be divided, then the agent with higher welfare does not gain. By weakening less first into weak less first or restricted less first, that both focus on agents with a significant level of welfare, and employing consistency, a classical invariance property requiring that the share of the surplus of any agent remains unchanged if some other agents take their shares and leave, we provide two additional characterizations.

In the second part of the paper, we investigate allocation rules in the setting of transferable utility (TU) games that support constrained egalitarianism in the sense that they distribute any increment of the worth of the grand coalition according to $f C E$. Indeed, we show that a Pareto optimal single-valued solution supports $f^{C E}$ if and only if it satisfies aggregate monotonicity (Megiddo, 1974), a property requiring that no player suffers if only the grand coalition becomes richer, and bounded pairwise fairness, requiring that

\footnotetext{
${ }^{1}$ Moreno-Ternero and Roemer (2012) provide a concise exposition of these two conceptions of distributive justice.

${ }^{2}$ See Ju and Moreno-Ternero (2018) for a discussion of different levels of fairness for the allocation of goods.
} 
an increasing worth of the grand coalition is distributed according to less first. Finally, we concentrate on single-valued solutions that combine coalitional rationality with the Lorenz criterion to promote equality. More precisely, we show that on the domain of balanced games (Bondareva, 1963; Shapley, 1967) any egalitarian solution satisfying aggregate monotonicity supports $f^{C E}$. As a consequence, we obtain that the egalitarian solution of Dutta and Ray (1989) and the lexmax solution of Arin et al. (2003) support $f^{C E}$ on the domains of convex games ${ }^{3}$ (Shapley, 1971) and games with large cores ${ }^{4}$ (Sharkey, 1982), respectively.

The remainder of the paper is organized as follows. Section 2 contains some general preliminaries. In Section 3 we introduce $f^{C E}$ and study the relations with the equal sharing rule and the non-constrained egalitarian rule, representing resource and welfare egalitarianism, respectively. Section 4 presents the axiomatic analysis of $f^{C E}$, including the logical independence of the properties. Section 5 is devoted to characterize, on some specific domains of TU games, the set of single-valued Pareto optimal solutions that support $f^{C E}$.

\section{Preliminaries}

Let $U$ be a set (the universe of potential agents) and $\mathcal{N}$ be the set of coalitions in $U$ (a coalition is a nonempty finite subset of $U$ ). Given $S, T \in \mathcal{N}$, we use $S \subset T$ to indicate strict inclusion, that is, $S \subseteq T$ and $S \neq T$. By $|S|$ we denote the cardinality of the coalition $S \in \mathcal{N}$. Given $N \in \mathcal{N}$, let $\mathbb{R}^{N}$ stand for the set of all real functions on $N$. An element $x \in \mathbb{R}^{N}, x=\left(x_{i}\right)_{i \in N}$, is a payoff vector for $N$. For all $S \subseteq N$, $x(S)=\sum_{i \in S} x_{i}$, with the convention $x(\emptyset)=0$. For each $x \in \mathbb{R}^{N}$ and $T \subseteq N, x_{T}$ denotes the restriction of $x$ to $T: x_{T}=\left(x_{i}\right)_{i \in T} \in \mathbb{R}^{T}$. Given $N \in \mathcal{N}$, for all $x, y \in \mathbb{R}^{N}$, $x \geq y$ if $x_{i} \geq y_{i}$ for all $i \in N$. For all $\alpha \in \mathbb{R}, \alpha_{+}=\max \{0, \alpha\}$. For any two vectors $y, x \in \mathbb{R}^{N}$ with $y(N)=x(N)$, we say that $y$ weakly Lorenz dominates $x$, denoted by $y \succeq_{\mathcal{L}} x$, if $\min \{y(S)|S \subseteq N| S \mid,=k\} \geq \min \{x(S)|S \subseteq N| S \mid,=k\}$, for all $k=1,2, \ldots, n-1$. We say that $y$ Lorenz dominates $x$, denoted by $y \succ_{\mathcal{L}} x$, if at least one of the above inequalities is strict. Given $x \in \mathbb{R}^{N}$, let $\mathcal{P}(x)=\left(N_{1}, N_{2}, \ldots, N_{k}\right)$ denote the ordered partition of $N$ that is determined by $N_{1}=\left\{i \in N \mid x_{i} \leq x_{j} \forall j \in N\right\}$ and $N_{m}=\left\{i \in N \backslash \bigcup_{j=1}^{m-1} N_{j} \mid x_{i} \leq x_{j} \forall j \in N \backslash \bigcup_{j=1}^{m-1} N_{j}\right\}$ for all $m=2, \ldots, k$.

\footnotetext{
${ }^{3}$ Outside the class of convex games, the existence of the egalitarian solution of Dutta and Ray (1989) is not guaranteed.

${ }^{4}$ The lexmax solution is defined for balanced games but, as we will see, in the whole domain of balanced games it does not support $f^{C E}$.
} 


\section{The constrained egalitarian rule}

A surplus-sharing problem is a triple $(N, x, t)$ where $N \in \mathcal{N}$ is the set of agents, $x \in$ $\mathbb{R}^{N}$ is the status quo or reference point, and $t \geq 0$ the surplus in terms of money. ${ }^{5}$ A surplus-sharing rule distributes the amount $t$ among the members of $N$ that are differentiated by $x \in \mathbb{R}^{N}$ which, depending on the situation, can denote the vector of individual opportunity costs or endowments of the agents or other objective references. Formally, it is a function $f$ that assigns to each surplus-sharing problem $(N, x, t)$ a vector $f(N, x, t) \in \mathbb{R}^{N}$ satisfying $\sum_{i \in N} f_{i}(N, x, t) \leq t$ (feasibility). ${ }^{6}$

Let $\mathcal{F}$ denote the set of all surplus-sharing rules with a finite set of agents in $\mathcal{N}$. We say that $f \in \mathcal{F}$ is Pareto optimal $(\mathbb{P O})$ if, for any suplus-sharing problem $(N, x, t), y(N)=t$ where $y=f(N, x, t)$. Moreover, $f \in \mathcal{F}$ satisfies nonnegativity $(\mathbb{N N})$ if $f(N, x, t) \geq 0 \in$ $\mathbb{R}^{N}$ for any surplus-sharing problem $(N, x, t)$. NN implies that no agent transfers part of her status quo to others.

In the literature (see, for instance, Moulin, 1987; Young, 1988; Chun, 1989; Pfingsten, 1991; Pfingsten, 1998), several surplus-sharing rules have been established and characterized but none of them cares about diminishing inequalities of the arising ex-post allocations, that is, after the allocation process.

A well-known form of egalitarianism is resource egalitarianism. The equal sharing rule, defined by setting

$$
f_{i}^{E Q}(N, x, t)=\frac{t}{|N|}
$$

for all surplus-sharing problems $(N, x, t)$ and all $i \in N$, distributes the available resource equally among the agents ignoring the initial status quo. Clearly, $f^{E Q}$ weakly Lorenz dominates every other Pareto optimal rule $f \in \mathcal{F}$, i.e., $f^{E Q}(N, x, t) \succeq_{\mathcal{L}} f(N, x, t)$ for any surplus-sharing problem $(N, x, t)$. However, it is easy to find instances (see Example 1) of surplus-sharing problems and Pareto optimal rules $f \in \mathcal{F}$ where $x+f^{E Q}(N, x, t)$ is Lorenz dominated by $x+f(N, x, t)$.

Another form of egalitarianism is welfare egalitarianism. The non-constrained egalitarian rule defined by setting

$$
f_{i}^{E}(N, x, t)=\frac{x(N)+t}{|N|}-x_{i}
$$

for all surplus-sharing problems $(N, x, t)$ and all $i \in N$, equalizes the welfare of the agents ex-post. Note that $x+f^{E}(N, x, t) \succeq_{\mathcal{L}} x+f(N, x, t)$ for any Pareto optimal rule $f \in \mathcal{F}$

\footnotetext{
${ }^{5}$ Usually, in the definition of a surplus-sharing problem the condition $x \in \mathbb{R}_{+}^{N}$ is imposed. Here, we consider a more general class of problems in which no restriction on $x$ is required.

${ }^{6}$ Other models incorporate additional requirements in defining a surplus-sharing rule (see, for instance, Moulin, 1987).
} 
and any surplus-sharing problem $(N, x, t)$. However, $f^{E}$ may require transfers between agents, i.e., it does not satisfy $\mathbb{N}$. Hence, for small $t$ some agents may lose when $f^{E}$ is applied so that they prefer not to collaborate.

To reconcile welfare egalitarianism with individual self-interest, we introduce the constrained egalitarian surplus-sharing rule, denoted by $f^{C E}$, and show that the ex-post allocation $x+f^{C E}(N, x, t)$ weakly Lorenz dominates the final outcome $x+f(N, x, t)$, for any Pareto optimal nonnegative surplus-sharing rule $f \in \mathcal{F}$ and for any surplus-sharing problem $(N, x, t)$.

Definition 1. The constrained egalitarian surplus-sharing rule is defined by

$$
f_{i}^{C E}(N, x, t)=\left(\lambda-x_{i}\right)_{+} \text {for all } N \in \mathcal{N}, x \in \mathbb{R}^{N}, t \in \mathbb{R}_{+} \text {, and } i \in N
$$

where $\lambda \in \mathbb{R}$ is determined by $\sum_{k \in N}\left(\lambda-x_{k}\right)_{+}=t$.

Thus, $f^{C E}$ treats equals (w.r.t. the status quo) equally, and makes unequal agents equal as far as this is possible. That is, it distributes the surplus to the poorer agents so that their payoffs become equal but not larger than the remaining agents' status quo payoffs. Note that $f^{C E}$ imposes egalitarianism constrained to each agent preserving her initial status quo. From the fact that $f^{E}(N, x, t)=f^{C E}(N, x, t)$ whenever $f^{E}(N, x, t) \geq 0$, it follows that

$$
f^{E}(N, x, t)=f^{C E}\left(N, x, t+t^{\prime}\right)-f^{E Q}\left(N, x, t^{\prime}\right),
$$

for any $t^{\prime} \geq 0$ such that $f^{E}\left(N, x, t+t^{\prime}\right) \geq 0$.

The following remark concerning $f^{C E}$ explains how to calculate $\lambda$ for any $x \in \mathbb{R}^{N}$ and $t>0$, and it will be useful in our proofs.

Remark 1. Let $N \in \mathcal{N}, x \in \mathbb{R}^{N}, t>0$, and $\lambda$ be such that $f_{i}^{C E}(N, x, t)=\left(\lambda-x_{i}\right)_{+}$ for all $i \in N$. Choose $i_{1}, \ldots, i_{n}$, where $n=|N|$, such that $\left\{i_{1}, \ldots, i_{n}\right\}=N$ and $x_{i_{1}} \leq$ $\cdots \leq x_{i_{n}}$. For $k \in\{1, \ldots, n\}$ define $\alpha_{k}(t)=\alpha_{k}=x\left(\left\{i_{1}, \ldots, i_{k}\right\}\right)-k x_{i_{k}}+t$ and observe that $\alpha_{1}=t>0$ and, for $k<n, \alpha_{k}-\alpha_{k+1}=k\left(x_{i_{k+1}}-x_{i_{k}}\right)$, hence $\alpha_{1} \geq \cdots \geq \alpha_{n}$. Now, with $k_{0}=\max \left\{k \in\{1, \ldots, n\} \mid \alpha_{k}>0\right\}$, we get

$$
\lambda=\frac{\alpha_{k_{0}}}{k_{0}}+x_{i_{k_{0}}}=\frac{x\left(\left\{i_{1}, \ldots, i_{k_{0}}\right\}\right)+t}{k_{0}} .
$$

Hence, $\lambda=x_{i_{k}}+f_{i_{k}}^{C E}(N, x, t)<x_{i_{k^{\prime}}}=x_{i_{k^{\prime}}}+f_{i_{k^{\prime}}}^{C E}(N, x, t)$, for all $k=1, \ldots, k_{0}$ and all $k^{\prime}=k_{0}+1, \ldots, n$.

Let us provide an example to illustrate the aforementioned surplus-sharing rules. 
Example 1. Consider the surplus-sharing problem defined by the set of agents $N=$ $\{1,2,3,4\}$, the status quo $x=(1,3,8,0)$, and $t=8$. It is not difficult to check that

$$
f^{E Q}(N, x, 8)=(2,2,2,2) \text { and } f^{E}(N, x, 8)=(4,2,-3,5) .
$$

To calculate $f^{C E}$, according to Remark $1, i_{1}=4$ and $i_{j}=j-1$ for $j=2,3,4$, and

$$
\begin{array}{lll}
\alpha_{1}=x_{i_{1}}-1 x_{i_{1}}+8 & =8 \\
\alpha_{2}=x_{i_{1}}+x_{i_{2}}-2 x_{i_{2}}+8 & =7, \\
\alpha_{3}=x_{i_{1}}+x_{i_{2}}+x_{i_{3}}-3 x_{i_{3}}+8 & = & 3, \\
\alpha_{4}=x_{i_{1}}+x_{i_{2}}+x_{i_{3}}+x_{i_{4}}-4 x_{i_{4}}+8 & = & -12 .
\end{array}
$$

Thus, $k_{0}=\max \left\{k \in\{1,2,3,4\} \mid \alpha_{k}>0\right\}=3, \lambda=\frac{\alpha_{3}}{3}+x_{i_{3}}=4$, and

$$
\begin{aligned}
& f_{1}^{C E}(N,(1,3,8,0), 8)=(4-1)_{+}=3 \\
& f_{2}^{C E}(N,(1,3,8,0), 8)=(4-3)_{+}=1 \\
& f_{3}^{C E}(N,(1,3,8,0), 8)=(4-8)_{+}=0 \\
& f_{4}^{C E}(N,(1,3,8,0), 8)=(4-0)_{+}=4 .
\end{aligned}
$$

Hence,

$$
f^{C E}(N, x, 8)=(3,1,0,4)
$$

Clearly,

$$
f^{E Q}(N, x, 8) \succ_{\mathcal{L}} f^{E}(N, x, 8) \text { and } f^{E Q}(N, x, 8) \succ_{\mathcal{L}} f^{C E}(N, x, 8) .
$$

Observe, however, that

$$
x+f^{C E}(N, x, 8)=(4,4,8,4) \succ_{\mathcal{L}}(3,5,10,2)=x+f^{E Q}(N, x, 8),
$$

and $x+f^{E}(N, x, 8)=(5,5,5,5)$ Lorenz dominates both distributions. Nevertheless, under $f^{E}$, agent 3 has no incentive to cooperate since $f_{3}^{E}(N, x, 8)=-3$.

We now show that, in general, among the nonnegative Pareto optimal surplus-sharing rules, when applied to any surplus-sharing problem, $f^{C E}$ yields the most egalitarian ex-post allocation.

Lemma 1. For all $N \in \mathcal{N}$, all $x \in \mathbb{R}^{N}$, and all $t \in \mathbb{R}_{+}$,

$$
x+f^{C E}(N, x, t) \succ_{L} x+z,
$$

where $z \in \mathbb{R}_{+}^{N}, z(N)=t$, and $z \neq f^{C E}(N, x, t)$.

Proof. Let $i_{1}, \ldots, i_{n}, k_{0}$, and $\lambda$ be defined as in Remark 1, $y=x+f^{C E}(N, x, t)$, and $y^{\prime}=x+z$. Let $\left\{j_{1}, \ldots, j_{k_{0}}\right\}=\left\{i_{1}, \ldots, i_{k_{0}}\right\}$ such that $y_{j_{1}}^{\prime} \leq \cdots \leq y_{j_{k_{0}}}^{\prime}$. Moreover, let $j_{k}=i_{k}$ for $k=\left\{k_{0}+1, \ldots, n\right\}$. Then, for each $k \in\{1, \ldots, n\}, \min \left\{y^{\prime}(S)|S \subseteq N| S \mid,=\right.$ 
$k\} \leq y^{\prime}\left(\left\{j_{1}, \ldots, j_{k}\right\}\right)$. Moreover, as $y_{i_{j}}=x_{i_{j}}$ for all $j \in\left\{k_{0}+1, \ldots, n\right\}$, by nonnegativity of $z$ we have $y_{i_{j}}^{\prime} \geq y_{i_{j}}$ so that, by $y^{\prime}(N)=y(N)$ and $y_{j_{1}}=\cdots=y_{j_{k_{0}}}=\lambda$ we conclude that $y^{\prime}\left(\left\{j_{1}, \ldots, j_{k}\right\}\right) \leqslant y\left(\left\{i_{1}, \ldots, i_{k}\right\}\right)$ for all $k \in\{1, \ldots, n\}$. Finally, as $z \neq f^{C E}(N, x, t)$, there is $k \in\{1, \ldots, n\}$ such that $y_{i_{k}} \neq y_{j_{k}}^{\prime}$ so that $y^{\prime}\left(\left\{j_{1}, \ldots, j_{k_{1}}\right\}\right)<y\left(\left\{i_{1}, \ldots, i_{k_{1}}\right\}\right)$ where $k_{1}$ is minimal in $\{1, \ldots, n\}$ such that $y_{i_{k_{1}}} \neq y_{j_{k_{1}}}^{\prime}$. Hence, $y \succ_{\mathcal{L}} y^{\prime}$.

Lemma 1 has the following immediate consequence.

Corollary 1. Let $f \in \mathcal{F}$ be a surplus-sharing rule that satisfy $\mathbb{N} \mathbb{N}$ and $\mathbb{P O}$. Then,

$$
x+f^{C E}(N, x, t) \succeq_{L} x+f(N, x, t),
$$

for all $N \in \mathcal{N}$, all $x \in \mathbb{R}^{N}$, and all $t \in \mathbb{R}_{+} \cdot{ }^{7}$

\section{Axiomatic analysis of $f^{C E}$}

In this section, we provide several axiomatizations of $f^{C E}$ either for fixed or variable sets of agents. Although the properties are stated for variable sets of agents (i.e., for surplussharing problems $(N, x, t)$ such that $N \in \mathcal{N})$, except for consistency, the remaining properties may be formulated for a fixed society $N \in \mathcal{N}$ of agents.

\subsection{Properties}

Together with $\mathbb{N N}$ and $\mathbb{P O}$, already defined in Section 3, we will use the following additional properties. A surplus-sharing rule $f \in \mathcal{F}$ satisfies

- Equal treatment of equals $(\mathbb{E} \mathbb{T})$ if for all $N \in \mathcal{N}$, all $x \in \mathbb{R}^{N}$, all $t \in \mathbb{R}_{+}$, and all $i, j \in N$, if $x_{i}=x_{j}$ then $f_{i}(N, x, t)=f_{j}(N, x, t)$;

- Resource monotonicity $(\mathbb{R M})$ if for all $N \in \mathcal{N}$, all $x \in \mathbb{R}^{N}$, and all $t, t^{\prime} \in \mathbb{R}_{+}$with $t^{\prime}>t, f\left(N, x, t^{\prime}\right) \geq f(N, x, t)$

- Path independence $(\mathbb{P I})$ if for all $N \in \mathcal{N}$, all $x \in \mathbb{R}^{N}$, and all $t, t^{\prime} \geq 0, f(N, x, t+$ $\left.t^{\prime}\right)=f(N, x, t)+f\left(N, x+f(N, x, t), t^{\prime}\right)$.

$\mathbb{E} \mathbb{T}$ is a simple equity requirement which imposes that equal agents (w.r.t. the status quo) should receive the same amount of the resource. $\mathbb{R M}$ is a solidarity condition requiring that nobody is worse off when there is more to be divided. Moulin (1987) introduces $\mathbb{P I}$, which requires that, regardless of the partition of the total amount of resource to

\footnotetext{
${ }^{7}$ The difference between Lemma 1 and Corollary 1 is that, for a particular surplus-sharing problem $(N, x, t)$, although $f \neq f^{C E}$, it could happen that $f(N, x, t)=f^{C E}(N, x, t)$.
} 
be allocated, its distribution may be dynamically obtained step-by-step by applying the surplus-sharing rule consecutively to the given elements of the partition, and taking into consideration the new status quo that emerges after the allocation process in the previous step.

Remark 2. Note that $\mathbb{P I}$ and $\mathbb{N N}$ imply $\mathbb{R M}$. Moreover, if $f \in \mathcal{F}$ satisfies $\mathbb{R M}$ and $\mathbb{P O}$, then, for all $N \in \mathcal{N}$ and all $x \in \mathbb{R}^{N}, f(N, x, \cdot): \mathbb{R}_{+} \rightarrow \mathbb{R}_{+}^{N}$ is a continuous mapping.

We now present three properties that require to prioritize agents with a lower status quo. A surplus-sharing rule $f \in \mathcal{F}$ satisfies

- Less first $(\mathbb{L} \mathbb{F})$ if for all $N \in \mathcal{N}$, all $x \in \mathbb{R}^{N}$, all $t \in \mathbb{R}_{+}$, and all $i, j \in N, i \neq j$, $f_{i}(N, x, t)>0$ implies $x_{i}-x_{j}<t$;

- Weak less first $(\mathbb{W} \mathbb{L} \mathbb{F})$ if for all $N \in \mathcal{N}$, all $x \in \mathbb{R}^{N}$, all $t \in \mathbb{R}_{+}$, and all $i, j \in N$, $i \neq j, f_{i}(N, x, t)>0$ and $x_{i}-x_{j} \geq t$ imply $f_{j}(N, x, t) \geq t$

- Restricted less first $(\mathbb{R L} \mathbb{F})$ if for all $N \in \mathcal{N}$, all $x \in \mathbb{R}^{N}$, all $t \in \mathbb{R}_{+}$, and all $i, j \in N$, $i \neq j$, with $x_{i} \geq x_{k}$ for all $k \in N, f_{i}(N, x, t)>0$ implies $x_{i}-x_{j}<t$.

$\mathbb{L} \mathbb{F}$ applies to any pair of agents, and it requires that an agent does not gain if her status quo exceeds the status quo of another agent by the surplus, while $\mathbb{W} \mathbb{L} \mathbb{F}$ imposes that the richest agent in the pair can only gain if the poorest agent in the pair receives at least the total surplus. $\mathbb{R L F}$ imposes $\mathbb{L F}$ only to pairs of agents containing an agent with the highest status quo. Similar protective properties for those agents with small "initial starting point" have been used in different models. Instances are No Domination (Moreno-Ternero and Roemer, 2012), in a model of resource allocation where agents are capable to transform wealth into non-transferable outcomes, or ex-ante fairness (Timoner and Izquierdo, 2016), in a context of rationing problems with ex-ante conditions. Observe that $\mathbb{L} \mathbb{F}$ implies $\mathbb{W} \mathbb{F}$ and $\mathbb{R} \mathbb{E}$. Furthermore, we show that $\mathbb{W} \mathbb{E}$ and $\mathbb{N}$ imply $\mathbb{L} \mathbb{F}$.

Proposition 1. If a surplus-sharing rule satisfies $\mathbb{N N}$ and $\mathbb{W} \mathbb{L}$ then also $\mathbb{L} \mathbb{F}$.

Proof. Let $f$ be a surplus-sharing rule satisfying $\mathbb{N N}$ and $\mathbb{W} \mathbb{L} \mathbb{F}$. If $x_{i}-x_{j} \geq t$ and $f_{i}(N, x, t)>0$ then, by $\mathbb{W} \mathbb{L} \mathbb{F}, f_{j}(N, x, t) \geq t$. By $\mathbb{N N}, \sum_{k \in N} f_{k}(N, x, t)>t$, which contradicts feasibility.

Making use of Remark 2, we now show that a surplus-sharing rule satisfying $\mathbb{L} \mathbb{F}, \mathbb{P O}$, $\mathbb{N}$, and $\mathbb{P I}$ also satisfies $\mathbb{E} \mathbb{T}$.

Proposition 2. If a surplus-sharing rule satisfies $\mathbb{P O}, \mathbb{N}, \mathbb{P I}$, and $\mathbb{L} \mathbb{F}$ then also $\mathbb{E} \mathbb{T}$. 
Proof. Let $N \in \mathcal{N}, x \in \mathbb{R}^{N}, t \in \mathbb{R}_{+}$, and $i, j \in N, i \neq j$, such that $x_{i}=x_{j}$. Let $f \in \mathcal{F}$ satisfy $\mathbb{P O}, \mathbb{N} \mathbb{N}, \mathbb{P} \mathbb{I}$ and $\mathbb{L} \mathbb{F}$.

If $t=0$, then by $\mathbb{P O}$ and $\mathbb{N N}, f_{i}(N, x, t)=f_{j}(N, x, t)=0$.

If $t>0$ suppose, w.l.o.g., $f_{i}(N, x, t)<f_{j}(N, x, t)$. Note that by $\mathbb{N} \mathbb{N}, f_{j}(N, x, t)>0$. Moreover, since $\mathbb{N N}$ and $\mathbb{P I}$ imply $\mathbb{R M}$, for all $0 \leq t^{\prime} \leq t$ we have that $f\left(N, x, t^{\prime}\right) \leq$ $f(N, x, t)$. By continuity and $\mathbb{R M}$ of $f$ (see Remark 2$), t^{*}=\min \left\{\tau \in \mathbb{R}_{+} \mid f_{j}(N, x, \tau)=\right.$ $\left.f_{j}(N, x, t)\right\}$ exists and, as $f_{j}\left(N, x, t^{*}\right)>f_{i}\left(N, x, t^{*}\right)$, for each $0<\hat{t}<t^{*}$ close enough to $t^{*}, f_{j}(N, x, \hat{t})-f_{i}(N, x, \hat{t})>t^{*}-\hat{t}$. As $x_{i}=x_{j}$, we obtain $t^{*}-\hat{t}<x_{j}+f_{j}(N, x, \hat{t})-$ $\left(x_{i}+f_{i}(N, x, \hat{t})\right)$. Hence, by $\mathbb{L} \mathbb{F}$ and $\mathbb{N N}, f_{j}\left(N, x+f(N, x, \hat{t}), t^{*}-\hat{t}\right)=0$. But then, by $\mathbb{P I}, f_{j}\left(N, x, t^{*}\right)=f_{j}(N, x, \hat{t})$ which means that $f_{j}(N, x, t)=f_{j}(N, x, \hat{t})$, contradicting the minimality of $t^{*}$.

Remark 3. Let us stress that $\mathbb{P O}, \mathbb{N N}, \mathbb{R M}$, and $\mathbb{L F}$ together are not enough to guarantee $\mathbb{E} \mathbb{T}$. Indeed, select $i \in U$ and define $f \in \mathcal{F}$ as follows. Let $N \in \mathcal{N}, x \in \mathbb{R}^{N}$, and $t \geq 0$. If $i \notin N$ or $i \in N$ and $x_{i}>x_{j}$ for some $j \in N \backslash\{i\}$, define $f(N, x, t)=f^{C E}(N, x, t)$. If $i \in N$ and $x_{i} \leq x_{j}$ for all $j \in N$, define $f_{i}(N, x, t)=t$ and $f_{j}(N, x, t)=0$ for all $j \in N \backslash\{i\}$. Then, $f$ satisfies $\mathbb{P O}, \mathbb{N N}, \mathbb{R M}$, and $\mathbb{L} \mathbb{F}$ but not $\mathbb{E} \mathbb{T}$.

Finally, we introduce consistency, a classical stability requirenment that forces the solution to coincide in both the original and the reduced surplus-sharing problem that results when some agents leave. Conditional consistency is a weakening of consistency that applies only if what is left to share among the agents in the reduced problem is nonnegative. A surplus-sharing rule $f \in \mathcal{F}$ satisfies

- Consistency $(\mathbb{C O})$ if for all $N \in \mathcal{N}$, all $x \in \mathbb{R}^{N}$, all $t \in \mathbb{R}_{+}$, and all $\emptyset \neq S \subset N$, $t-\sum_{i \in N \backslash S} f_{i}(N, x, t) \geq 0$ and $f_{S}(N, x, t)=f\left(S, x_{S}, t-\sum_{i \in N \backslash S} f_{i}(N, x, t)\right)$.

- Conditional consistency $(\mathbb{C} \mathbb{C O})$ if for all $N \in \mathcal{N}$, all $x \in \mathbb{R}^{N}$, all $t \in \mathbb{R}_{+}$, and all $\emptyset \neq S \subset N$, the following condition holds: if $t-\sum_{i \in N \backslash S} f_{i}(N, x, t) \geq 0$, then $f_{S}(N, x, t)=f\left(S, x_{S}, t-\sum_{i \in N \backslash S} f_{i}(N, x, t)\right)$.

From $\mathbb{P O}$ and $\mathbb{N}$, we have $t-\sum_{i \in N \backslash S} f_{i}(N, x, t)=\sum_{i \in S} f_{i}(N, x, t) \geq 0$, for any surplussharing problem $(N, x, t)$. Thus, under $\mathbb{P O}$ and $\mathbb{N}, \mathbb{C} \mathbb{C O}$ implies $\mathbb{C} \mathbb{O}$. Bilateral conditional consistency $(2-\mathbb{C} \mathbb{O})$ requires $\mathbb{C} \mathbb{C O}$ for reduced surplus-sharing problems with two agents, i.e., $|S|=2$.

\subsection{Characterizations with and without consistency}

First, we deal with a fixed agent set $N \in \mathcal{N}$. By definition, $f^{C E}$ satisfies $\mathbb{P O}$ and $\mathbb{N}$. 
Proposition 3. The surplus-sharing rule $f^{C E}$ satisfies $\mathbb{P I}$ and $\mathbb{L} \mathbb{F}$.

Proof. Let $x \in \mathbb{R}^{N}$ and $t \geq 0$.

To show $\mathbb{P I}$, let $i_{1}, \ldots, i_{n}$ be defined as in Remark $1, t=t_{1}+t_{2}, t_{1}, t_{2}>0$,

$$
k_{0}^{1}=\max \left\{k \in\{1, \ldots, n\} \mid x\left(\left\{i_{1}, \ldots, i_{k}\right\}\right)+t_{1}>k x_{i_{k}}\right\}
$$

and

$$
k_{0}=\max \left\{k \in\{1, \ldots, n\} \mid x\left(\left\{i_{1}, \ldots, i_{k}\right\}\right)+t>k x_{i_{k}}\right\} .
$$

That is, with

$$
\lambda_{1}=\frac{x\left(\left\{i_{1}, \ldots, i_{k_{0}^{1}}\right\}\right)+t_{1}}{k_{0}^{1}} \text { and } \lambda=\frac{x\left(\left\{i_{1}, \ldots, i_{k_{0}}\right\}\right)+t}{k_{0}},
$$

we have $f_{i}^{C E}\left(N, x, t_{1}\right)=\left(\lambda_{1}-x_{i}\right)_{+}$and $f_{i}^{C E}(N, x, t)=\left(\lambda-x_{i}\right)_{+}$, for all $i \in N$. Let $y=x+f^{C E}\left(N, x, t_{1}\right)$. By Remark $1, y_{i_{1}}=\cdots=y_{i_{0}^{1}}<y_{i_{k_{0}^{1}+1}} \leq \cdots \leq y_{i_{n}}$ and $k_{0}^{1} \leq k_{0}$. As $k_{0} \lambda-x\left(\left\{i_{1}, \ldots, i_{k_{0}}\right\}\right)=t$ and $k_{0}^{1} \lambda_{1}-x\left(\left\{i_{1}, \ldots, i_{k_{0}^{1}}\right\}\right)=t_{1}$, we conclude that

$$
\begin{aligned}
k_{0} \lambda-y\left(\left\{i_{1}, \ldots, i_{k_{0}}\right\}\right) & =k_{0}\left(\lambda-\lambda_{1}\right)-x\left(\left\{i_{k_{0}^{1}+1}, \ldots, i_{k_{0}}\right\}\right) \\
& =k_{0} \lambda-x\left(\left\{i_{1}, \ldots, i_{k_{0}}\right\}\right)+x\left(\left\{i_{1}, \ldots, i_{k_{0}^{1}}\right\}\right)-k_{0}^{1} \lambda_{1} \\
& =t-t_{1}=t_{2}
\end{aligned}
$$

so that $\mathbb{P I}$ is shown.

To show $\mathbb{L} \mathbb{F}$, suppose there are $i, j \in N, i \neq j$, with $x_{i}-x_{j} \geq t$ and $f_{i}^{C E}(N, x, t)>0$. Since $x_{i} \geq x_{j}, f_{i}^{C E}(N, x, t) \leq f_{j}^{C E}(N, x, t)$ and thus $f_{j}^{C E}(N, x, t)>0$. This means that $x_{i}+f_{i}^{C E}(N, x, t)=x_{j}+f_{j}^{C E}(N, x, t)$ (see Remark 1), which implies $x_{i}-x_{j}=$ $f_{j}^{C E}(N, x, t)-f_{i}^{C E}(N, x, t) \geq t$. But then $f_{j}^{C E}(N, x, t)>t$, contradicting $\mathbb{P} \mathbb{O}$. Hence, $f_{i}^{C E}(N, x, t)=0$.

Our first characterization result imposes $\mathbb{P O}, \mathbb{N N}, \mathbb{P I}$, and $\mathbb{L} \mathbb{F}$.

Theorem 1. The unique surplus-sharing rule that satisfies $\mathbb{P O}, \mathbb{N N}, \mathbb{P I}$, and $\mathbb{L} \mathbb{F}$ is $f^{C E}$.

Proof. $f^{C E}$ satisfies $\mathbb{P O}$ and $\mathbb{N N}$ and, by Proposition $3, \mathbb{P I}$ and $\mathbb{L} \mathbb{F}$.

For the uniqueness part, let $f$ be a surplus-sharing rule that satisfies the desired axioms, hence also $\mathbb{E} \mathbb{T}$ by Proposition 2 . Let $(N, x, t)$ be a surplus-sharing problem. It remains to show that $f(N, x, t)=f^{C E}(N, x, t)$. We proceed by induction on $m(x)=\left|\left\{x_{i} \mid i \in N\right\}\right|$. If $m(x)=1$, then the proof is finished by $\mathbb{E} \mathbb{T}$ and $\mathbb{P O}$. Our inductive hypothesis is that $f(N, x, t)=f^{C E}(N, x, t)$ whenever $m(x)<k$ for some $k \in \mathbb{N}, k>1$. Now, assume that $m(x)=k$. Let $S(x)=S=\left\{i \in N \mid x_{i} \leq x_{j}\right.$ for all $\left.j \in N\right\}, \alpha(x)=\alpha=\min _{i \in N} x_{i}$, and 
$\beta(x)=\beta=\min _{i \in N \backslash S} x_{i}$. Let $|S|=s$. By $\mathbb{E T}, f_{i}(N, x, t)=f_{j}(N, x, t)$ for all $i \in S$. We distinguish two cases:

Case 1: $t \leq s(\beta-\alpha)$. By $\mathbb{P O}$ and $\mathbb{N N}$ it remains to show that $f_{i}(N, x, t)=t / s$ for all $i \in S$. Assume the contrary. As $f(N, x, 0)=f^{C E}(N, x, 0)=0 \in \mathbb{R}^{N}$ by $\mathbb{N}$, continuity (see Remark 2) of $f(N, x, \cdot)$ implies that, for all $i \in S$,

$$
t^{\prime}=\max \left\{\tilde{t} \in \mathbb{R} \mid 0 \leq \tilde{t} \leq t, f_{i}(N, x, \tilde{t})=f_{i}^{C E}(N, x, \tilde{t})\right\}
$$

exists and, by our assumption, $t^{\prime}<t$. Let $x^{\prime}=x+f\left(N, x, t^{\prime}\right)$. Note that $S\left(x^{\prime}\right)=$ $S, \beta\left(x^{\prime}\right)=\beta$, and $\alpha\left(x^{\prime}\right)=\alpha(x)+t^{\prime} / s$. Now, for any $0<t^{\prime \prime}<\left(\beta-\alpha\left(x^{\prime}\right)\right) / s, f_{j}\left(N, x^{\prime}, t^{\prime \prime}\right)=$ 0 for all $j \in N \backslash S$ by $\mathbb{L} \mathbb{F}$ and $\mathbb{N N}$ so that $f\left(N, x^{\prime}, t^{\prime \prime}\right)=f^{C E}\left(N, x^{\prime}, t^{\prime \prime}\right)$ by $\mathbb{E} \mathbb{T}$ and $\mathbb{P} \mathbb{O}$. Therefore, by $\mathbb{P I}$ of $f$ and $f^{C E}, f\left(N, x, t^{\prime}+t^{\prime \prime}\right)=f\left(N, x, t^{\prime}\right)+f\left(N, x^{\prime}, t^{\prime \prime}\right)=f^{C E}\left(N, x, t^{\prime}\right)+$ $f^{C E}\left(N, x^{\prime}, t^{\prime \prime}\right)=f^{C E}\left(N, x, t^{\prime}+t^{\prime \prime}\right)$, which contradicts the maximality of $t^{\prime}$.

Case 2: $t>s(\beta-\alpha)=t^{\prime}$. By Case 1, $f\left(N, x, t^{\prime}\right)=f^{C E}\left(N, x, t^{\prime}\right)$. Let $x^{\prime}=x+$ $f\left(N, x, t^{\prime}\right)$. Then $m\left(x^{\prime}\right)=m(x)-1$ so that, by the inductive hypothesis, $f\left(N, x^{\prime}, t-t^{\prime}\right)=$ $f^{C E}\left(N, x^{\prime}, t-t^{\prime}\right)$. Finally, by $\mathbb{P I}$ we receive $f(N, x, t)=f\left(N, x, t^{\prime}\right)+f\left(N, x^{\prime}, t-t^{\prime}\right)=$ $f^{C E}\left(N, x, t^{\prime}\right)+f^{C E}\left(N, x^{\prime}, t-t^{\prime}\right)=f^{C E}(N, x, t)$.

By Proposition $1, \mathbb{N} \mathbb{N}$ and $\mathbb{W} \mathbb{F}$ are equivalent to $\mathbb{N}$ and $\mathbb{L} \mathbb{F}$. Thus, $\mathbb{L} \mathbb{F}$ in Theorem 1 may be replaced by $\mathbb{W} \mathbb{L F}$ so that we receive the following result.

Corollary 2. The unique surplus-sharing rule that satisfies $\mathbb{P O}, \mathbb{N N}, \mathbb{P I}$, and $\mathbb{W} \mathbb{F}$, is $f^{C E}$.

It is insightful to remark that $f^{E Q}$ satisfies all properties in Theorem 1 except $\mathbb{L} \mathbb{F}$, making $\mathbb{L} \mathbb{F}$ the key property for $f^{C E}$. Moreover, as $f^{E}$ satisfies all properties in Corollary 2 except $\mathbb{N N}$, this property becomes essential for distinguishing $f^{C E}$ from $f^{E}$. Note that neither $f^{E Q}$ satisfies $\mathbb{W} \mathbb{L} \mathbb{F}$ nor $f^{E}$ satisfies $\mathbb{L} \mathbb{F}$.

Now, we consider a variable society of agents. Our third characterization result replaces $\mathbb{L} \mathbb{F}$ in Theorem 1 by $\mathbb{R L F}$ and 2-CCCO .

Theorem 2. The unique surplus-sharing rule that satisfies $\mathbb{P O}, \mathbb{N}, \mathbb{P I}, \mathbb{R L} \mathbb{F}$, and 2$\mathbb{C} \mathbb{C O}$ is $f^{C E}$.

Proof. $f^{C E}$ satisfies $\mathbb{P O}, \mathbb{N N}, \mathbb{P I}$, and $\mathbb{R} \mathbb{L} \mathbb{F}$. To show 2-CCCO , let $N \in \mathcal{N}, x \in \mathbb{R}^{N}, t \geq 0$, and $\emptyset \neq S \subset N$, then $t^{\prime}=t-\sum_{i \in N \backslash S} f_{i}(N, x, t)=\sum_{i \in S} f_{i}(N, x, t) \geq 0$ by $\mathbb{P O}$ and $\mathbb{N}$. Let $y=f^{C E}(N, x, t)$ and $z=\left(f^{C E}\left(S, x_{S}, t^{\prime}\right), y_{N \backslash S}\right)$. By Corollary $1, x+y \succeq_{\mathcal{L}} x+z$. As $y_{N \backslash S}=z_{N \backslash S}$, the definition of Lorenz domination yields $x_{S}+y_{S} \succeq_{\mathcal{L}} x_{S}+z_{S}=$ $x_{S}+f^{C E}\left(S, x_{S}, t^{\prime}\right)$. Finally, by Corollary $1, x_{S}+f^{C E}\left(S, x_{S}, t^{\prime}\right) \succeq_{\mathcal{L}} x_{S}+y_{S}$, so that we obtain $y_{S}=f^{C E}\left(S, x_{S}, t^{\prime}\right)$. Hence, $f^{C E}$ satisfies $\mathbb{C} \mathbb{O}$ and, consequently, 2-CCCO . 
For the uniqueness part, let $N \in \mathcal{N}, x \in \mathbb{R}^{N}, t \geq 0$, and $f$ be a surplus-sharing rule that satisfies the desired properties. By Theorem 1, it suffices to show that $f$ satisfies $\mathbb{L F}$. To this end, let $N \in \mathcal{N}$ with $|N| \geq 2, x \in \mathbb{R}^{N}$, and $t \geq 0$. If $i, j \in N$ such that $i \neq j$ and $f_{i}(N, x, t)>0$, we have, with $S=\{i, j\}$, by $\mathbb{P O}$ and $\mathbb{N N}, t^{\prime}=t-\sum_{k \in N \backslash S} f_{k}(N, x, t) \geq 0$ so that, by $2-\mathbb{C} \mathbb{C O}, f\left(S, x_{S}, t^{\prime}\right)=f_{S}(N, x, t)$. Hence, by $\mathbb{R L F}$ applied to $\left(S, x_{S}, t^{\prime}\right)$, $x_{i}-x_{j}<t^{\prime} \leq t$ and thus $\mathbb{L F}$ is shown.

As before, $\mathbb{R L F}$ distinguishes $f^{E Q}$ from $f^{C E}$ since $f^{E Q}$ also satisfies 2- $\mathbb{C} \mathbb{O}$. On the other hand, as $f^{E}$ meets $2-\mathbb{C} \mathbb{C O}$ and $\mathbb{R} \mathbb{L} \mathbb{F}$, the property of $\mathbb{N}$ is crucial again to compare $f^{C E}$ with $f^{E}$ from a normative point of view.

\subsection{Logical independence of the properties}

In this subsection, we show the non-redundancy of the properties in the above characterization results.

(i) Non-redundancy of the properties in Theorem 1, provided $|U| \geq 2$ :

- The equal sharing rule $f^{E Q}$ satisfies $\mathbb{P O}, \mathbb{N N}, \mathbb{P I}$ but not $\mathbb{L} \mathbb{F}$.

- Let $N \in \mathcal{N}, x \in \mathbb{R}^{N}$, and $t \geq 0$. Denote $N_{1}=\left\{i \in N \mid x_{i} \leq x_{j} \forall j \in N\right\}$. Define

$$
f_{i}^{\leq}(N, x, t)=\left\{\begin{array}{rll}
\frac{t}{\left|N_{1}\right|} & \text { if } & i \in N_{1}, \\
0 & \text { if } & i \in N \backslash N_{1} .
\end{array}\right.
$$

Then, $f \leq$ satisfies $\mathbb{P O}, \mathbb{N N}$, and $\mathbb{L} \mathbb{F}$ but not $\mathbb{P I}$.

- Let $N \in \mathcal{N}, x \in \mathbb{R}^{N}$, and $t \geq 0$. Define

$$
f^{0}=(0,0, \ldots, 0) \in \mathbb{R}^{N}
$$

Then, $f^{0}$ satisfies $\mathbb{N} \mathbb{N}, \mathbb{P I}$, and $\mathbb{L} \mathbb{F}$ but not $\mathbb{P} \mathbb{O}$.

- Let $N \in \mathcal{N}, x \in \mathbb{R}^{N}$, and $t \geq 0$. For $|N|=1$, put $f^{*}(N, x, t)=t$ for all $t \geq 0$.

Now consider the case $|N| \geq 2$. Let $\alpha(x)$ denote the second smallest component of $x$, i.e., $\alpha(x)=\min \{x(S)|S \subseteq N| S \mid,=2\}-\min _{i \in N} x_{i}$, and let $S=S(x)=$ $\left\{i \in N \mid x_{i} \leq \alpha(x)\right\}$, i.e., $S$ is the coalition of players whose payoffs belong to the 2 smallest status quo payoffs. Define $\tilde{x} \in \mathbb{R}^{N}$ by $\tilde{x_{S}}=x_{S}+f^{E}\left(S, x_{S}, 0\right)$, i.e., $\tilde{x}_{i}=x(S) /|S|$ for all $i \in S$, and $\tilde{x}_{N \backslash S}=x_{N \backslash S}$, i.e., $\tilde{x}_{j}=x_{j}$ for all $j \in N \backslash S$. Now, for each $t \geq 0$, put $f^{*}(N, x, t)=\tilde{x}-x+f^{C E}(N, \tilde{x}, t)$.

Hence, if $|N|=2, f^{*}(N, x, t)=f^{E}(N, x, t)$. 
Moreover, $\mathbb{P O}$ of $f^{*}$ is guaranteed by definition and the facts that $f^{E}$ and $f^{C E}$ satisfy this axiom.

Let $t, t^{\prime} \geq 0$. As with $y=\tilde{x}+f^{C E}(N, \tilde{x}, t)$ we have $\tilde{y}=y$, we deduce from $\mathbb{P I}$ of $f^{C E}$ that $f^{*}\left(N, x, t+t^{\prime}\right)=\tilde{x}-x+f^{C E}\left(N, \tilde{x}, t+t^{\prime}\right)=\tilde{x}-x+f^{C E}(N, \tilde{x}, t)+$ $f^{C E}\left(N, \tilde{x}+f^{C E}(N, \tilde{x}, t), t^{\prime}\right)=f^{*}(N, x, t)+f^{*}\left(N, \tilde{x}+f^{*}(\tilde{x}, t), t^{\prime}\right)$ so that $f^{*}$ also satisfies $\mathbb{P I}$.

In order to show $\mathbb{L} \mathbb{F}$, we assume $f_{i}^{*}(N, x, t)>0$ and $j \in N \backslash\{i\}$ with $x_{j} \leq x_{i}$. If $\tilde{x}=x$, then $x_{i}-x_{j}<t$ by $\mathbb{L F}$ of $f^{C E}$. Hence, we may assume that $x$ has a unique minimizer $k$. If $i=k, x_{i}-x_{j}<0 \leq t$. If $i \neq k$, then $x_{\ell}+f_{\ell}^{*}(N, x, t)=x_{i}+f_{i}^{*}(N, x, t)$ for all $\ell \in S(x)$ so that $x_{i}-x_{k}<t$. Hence, $f^{*}$ satisfies $\mathbb{L} \mathbb{F}$.

Thus, $f^{*}$ satisfies $\mathbb{P O}, \mathbb{P I}$, and $\mathbb{L} \mathbb{F}$ but not $\mathbb{N N}$.

(ii) Non-redundancy of the properties in Corollary 2, provided $|U| \geq 2$ :

$f^{E Q}$ satisfies $\mathbb{P O}, \mathbb{N N}$, and $\mathbb{P I}$ but not $\mathbb{W} \mathbb{E} ; f \leq$ satisfies $\mathbb{P O}, \mathbb{N N}$, and $\mathbb{W} \mathbb{L} \mathbb{F}$ but not $\mathbb{P I}$; $f^{E}$ satisfies $\mathbb{P O}, \mathbb{P I}$, and $\mathbb{W} \mathbb{E}$ but not $\mathbb{N N} ; f^{0}$ satisfies $\mathbb{N N}, \mathbb{P I}$, and $\mathbb{W} \mathbb{E}$ but not $\mathbb{P O}$.

(iii) Non-redundancy of the properties in Theorem 2, provided $|U| \geq 2$ :

$f^{E Q}$ satisfies $\mathbb{P O}, \mathbb{N N}, \mathbb{P I}$, and $2-\mathbb{C} \mathbb{C O}$ but not $\mathbb{R} \mathbb{L} ; f \leq$ satisfies $\mathbb{P O}, \mathbb{N N}, \mathbb{R L} \mathbb{F}$, and $2-\mathbb{C} \mathbb{O}$ but not $\mathbb{P I} ; f^{E}$ satisfies $\mathbb{P O}, \mathbb{P I}, \mathbb{R L} \mathbb{F}$, and $2-\mathbb{C} \mathbb{O}\left(\right.$ but not $\mathbb{N}$; $f^{0}$ satisfies $\mathbb{N}$, $\mathbb{P I}, \mathbb{R} \mathbb{L}$, and $2-\mathbb{C} \mathbb{C}($ but not $\mathbb{P O}$.

Let $N \in \mathcal{N}, x \in \mathbb{R}^{N}$, and $t \geq 0$. Define $\hat{f}(N, x, t)$ as follows: for all $i \in N$,

$$
\hat{f}_{i}(N, x, t)=\left\{\begin{aligned}
\frac{t}{n \gamma-x(N)}\left(\gamma-x_{i}\right) & \text { if } t<n \gamma-x(N), \\
f_{i}^{C E}(N, x, t) & \text { if } t \geq n \gamma-x(N)
\end{aligned}\right.
$$

where $n=|N|$ and $\gamma=\gamma(x)=\max _{i \in N} x_{i}$.

Clearly, $\hat{f}$ satisfies $\mathbb{P O}$ and $\mathbb{N N}$. To check $\mathbb{R} \mathbb{L} \mathbb{F}$, we assume $\hat{f}_{i}(N, x, t)>0$ such that $x_{i} \geq x_{j}$ for all $j \in N \backslash\{i\}$ so that $\gamma(x)=x_{i}$. Therefore, $\hat{f}_{i}(N, x, t)=f_{i}^{C E}(N, x, t)$ and so $\mathbb{R} \mathbb{L}$ follows from $\mathbb{L} \mathbb{F}$ of $f^{C E}$.

In order to prove $\mathbb{P I}$, let $t, t^{\prime} \geq 0$ and $i \in N$. We distinguish two cases:

Case 1: $t+t^{\prime}<n \gamma(x)-x(N)$. By definition of $\hat{f}$,

$$
\hat{f}_{i}\left(N, x, t+t^{\prime}\right)=\frac{t+t^{\prime}}{n \gamma(x)-x(N)}\left(\gamma(x)-x_{i}\right) \text { and } \hat{f}_{i}(N, x, t)=\frac{t}{n \gamma(x)-x(N)}\left(\gamma(x)-x_{i}\right) .
$$

Let $y=x+\hat{f}(N, x, t)$. Then, $\gamma(y)=\gamma(x), y(N)=x(N)+t$ and $n \gamma(y)-y(N)=$ $n \gamma(x)-x(N)-t>t^{\prime}$. Thus,

$$
\hat{f}_{i}\left(N, y, t^{\prime}\right)=\frac{t^{\prime}}{n \gamma(y)-y(N)}\left(\gamma(y)-y_{i}\right)
$$


Combining equations (12) and (13), we receive

$$
\hat{f}_{i}(N, x, t)+\hat{f}_{i}\left(N, y, t^{\prime}\right)=\frac{t+t^{\prime}}{n \gamma(x)-x(N)}\left(\gamma(x)-x_{i}\right)=\hat{f}_{i}\left(N, x, t+t^{\prime}\right) .
$$

Case 2: $t+t^{\prime} \geq n \gamma(x)-x(N)$. By definition of $f^{C E}, \hat{f}_{i}\left(N, x, t+t^{\prime}\right)=\frac{x(N)+t+t^{\prime}}{n}-x_{i}$. We distinguish two subcases:

Subcase 2.1: $t \geq n \gamma(x)-x(N)$. By definition, $\hat{f}_{i}(N, x, t)=\frac{x(N)+t}{n}-x_{i}$. Let $y=$ $x+\hat{f}(N, x, t)$. Then, $y_{i}=\frac{x(N)+t}{n}$, and since $\hat{f}$ satisfies $\mathbb{E} \mathbb{T}$, we have that $\hat{f}_{i}\left(N, y, t^{\prime}\right)=\frac{t^{\prime}}{n}$. Hence, $\hat{f}_{i}\left(N, x, t+t^{\prime}\right)=\frac{x(N)+t+t^{\prime}}{n}-x_{i}=\frac{x(N)+t}{n}-x_{i}+\frac{t^{\prime}}{n}=\hat{f}_{i}(N, x, t)+\hat{f}_{i}\left(N, y, t^{\prime}\right)$.

Subcase 2.2: $t<n \gamma(x)-x(N)$. By definition, $\hat{f}_{i}(N, x, t)=\frac{t}{n \gamma(x)-x(N)}\left(\gamma(x)-x_{i}\right)$. Let $y=x+\hat{f}(N, x, t)$. Then, $\gamma(y)=\gamma(x), y(N)=x(N)+t$, and $n \gamma(y)-y(N)=$ $n \gamma(x)-x(N)-t \leq t^{\prime}$. Hence,

$$
\begin{aligned}
\hat{f}_{i}\left(N, y, t^{\prime}\right) & =\frac{y(N)+t^{\prime}}{n}-x_{i}-\hat{f}_{i}(N, x, t) \\
& =\frac{x(N)+t+t^{\prime}}{n}-x_{i}-\hat{f}_{i}(N, x, t) \\
& =\hat{f}_{i}\left(N, x, t+t^{\prime}\right)-\hat{f}_{i}(N, x, t) .
\end{aligned}
$$

This concludes the proof of $\mathbb{P I}$. Since $\hat{f} \neq f^{C E}, \hat{f}$ does not satisfy $2-\mathbb{C} \mathbb{C} \mathbb{O}$.

\section{Game theoretical support of $f^{C E}$}

In this section, we investigate constrained welfare egalitarianism of single-valued solutions for certain classes of transferable utility games.

A transferable utility game, for short game, is a pair $(N, v)$ where $N \in \mathcal{N}$ and $v$ is a function that associates a real number $v(S)$ with each $S \subseteq N$. We asume that $v(\emptyset)=0$. For $t \in \mathbb{R}$ and any game $(N, v)$, denote by $\left(N, v^{t}\right)$ the game that differs from $(N, v)$ at most inasmuch as $v^{t}(N)=v(N)+t$. Let $\Gamma$ denote the set of all games. We often consider a domain of games that allow to increase the worth of the grand coalition. Thus, we say that $\Gamma^{\prime} \subseteq \Gamma$ is closed under increments if for all $(N, v) \in \Gamma^{\prime}$ and all $t>0,\left(N, v^{t}\right) \in \Gamma^{\prime}$. The set of feasible payoff vectors of $(N, v)$ is defined by $X^{*}(N, v)=\left\{x \in \mathbb{R}^{N} \mid x(N) \leq v(N)\right\}$, the set of Pareto optimal payoff vectors by $X(N, v)=\left\{x \in \mathbb{R}^{N} \mid x(N)=v(N)\right\}$, and the core by $C(N, v)=\{x \in X(N, v) \mid x(S) \geq v(S)$ for all $S \subseteq N\}$. A (single-valued) solution on a domain $\Gamma^{\prime} \subseteq \Gamma$ is a function $\sigma$ that associates with each $(N, v) \in \Gamma^{\prime}$ a unique element $\sigma(N, v)$ of $X^{*}(N, v)$. A solution $\sigma$ on $\Gamma^{\prime}$ satisfies

- Pareto optimality (PO) if for all $(N, v) \in \Gamma^{\prime}, \sigma(N, v) \in X(N, v)$;

- Aggregate monotonicity (Megiddo, 1974) (AM) if for all $(N, v) \in \Gamma^{\prime}$ and all $t>0$ such that $\left(N, v^{t}\right) \in \Gamma^{\prime}, \sigma\left(N, v^{t}\right) \geq \sigma(N, v)$. 
PO simply says that the worth of the grand coalition should be exhausted. AM means that every player should be better-off when the grand coalition becomes richer. In order to characterize the set of solutions that distribute an increment of the worth of the grand coalition according to $f^{C E}$, we introduce the following properties. A solution $\sigma$ on $\Gamma^{\prime}$ satisfies

- Weak continuity (WC) if for all $(N, v) \in \Gamma^{\prime}$ and all sequences $\left(\alpha_{k}\right)_{k \in \mathbb{N}}$ with limit $v(N)$ the following condition is satisfied: Let, for $k \in \mathbb{N},\left(N, v_{k}\right)$ be the game defined by $v_{k}(N)=\alpha_{k}$ and $v_{k}(S)=v(S)$ for all $S \subset N$. If $\left(N, v_{k}\right) \in \Gamma^{\prime}$ for all $k \in \mathbb{N}$ and if $\left(\sigma\left(N, v^{k}\right)\right)_{k \in \mathbb{N}}$ converges to some $x$, then $x=\sigma(N, v)$.

- Bounded pairwise fairness (BPF) if for all $(N, v) \in \Gamma^{\prime}$, all $t>0$ such that $\left(N, v^{t}\right) \in$ $\Gamma^{\prime}$, and all $i, j \in N, \sigma_{i}\left(N, v^{t}\right)-\sigma_{i}(N, v)>0$ implies $\sigma_{i}(N, v)-\sigma_{j}(N, v)<t$.

$\mathrm{PO}$ and AM together imply WC. The property BPF is a priority requirement imposing that, if the difference in payoffs between two players in the initial game $(N, v)$ exceeds the total additional amount $t$ to be divided, then in the game $\left(N, v^{t}\right)$ the originally richer player cannot be better off than before.

Definition 2. A solution $\sigma$ on $\Gamma^{\prime}$ is said to support constrained welfare egalitarianism if for all $(N, v) \in \Gamma^{\prime}$ and all $t>0$, whenever $\left(N, v^{t}\right) \in \Gamma^{\prime}$ it holds that

$$
\sigma\left(N, v^{t}\right)=\sigma(N, v)+f^{C E}(N, \sigma(N, v), t)
$$

A solution $\sigma$ that supports constrained welfare egalitarianism exhibits a dynamic behaviour in the sense that, on a sequence of games with increasing worth of the grand coalition, $\sigma$ evolves dynamically assigning an allocation in each period $k$ that is uniquely determined by the allocation in the previous period $k-1$, following the path recommended by $f^{C E}$.

Note that $\mathrm{AM}$ is implied by Equation (14) and the fact that $f^{C E}$ satisfies $\mathbb{N N}$. In order to show that a Pareto optimal solution satisfies AM and BPF if and only if it supports constrained welfare egalitarianism, the following lemma that resembles Proposition 2 is useful. While in the framework of surplus-sharing problems $\mathbb{P O}, \mathbb{N N}, \mathbb{R M}$, and $\mathbb{L} \mathbb{F}$ together do not imply $\mathbb{E} \mathbb{T}$ (see Remark 3), here PO, AM, and BPF are enough to ensure a kind of equal treatment property that only applies when the worth of the grand coalition increases.

Lemma 2. Let $\Gamma^{\prime} \subseteq \Gamma$ be closed under increments and $\sigma$ be a solution on $\Gamma^{\prime}$ that satisfies $\mathrm{PO}, \mathrm{AM}$, and $\mathrm{BPF}$. For all $(N, v) \in \Gamma^{\prime}$, all $i, j \in N$, and all $t \in \mathbb{R}_{+}$, if $\sigma_{i}(N, v)=\sigma_{j}(N, v)$, then $\sigma_{i}\left(N, v^{t}\right)=\sigma_{j}\left(N, v^{t}\right)$. 
Proof. Let $\sigma$ be a solution on $\Gamma^{\prime}$ satisfying PO, AM and BPF. Suppose, on the contrary, there exist $i, j \in N$ and $t>0$ such that $\sigma_{i}(N, v)=\sigma_{j}(N, v)$ but $\sigma_{j}\left(N, v^{t}\right)>\sigma_{i}\left(N, v^{t}\right)$. By PO and AM, $\sigma$ meets WC. Therefore, there exists a minimal $t^{*} \in(0, t]$ such that $\sigma_{j}\left(N, v^{t^{*}}\right)=\sigma_{j}\left(N, v^{t}\right)$. Hence,

$$
\sigma_{j}\left(N, v^{t^{\prime \prime}}\right)<\sigma_{j}\left(N, v^{t^{*}}\right)=\sigma_{j}\left(N, v^{t}\right) \text { for all } t^{\prime \prime} \in\left[0, t^{*}\right)
$$

Note that $\sigma_{j}(N, v)<\sigma_{j}\left(N, v^{t}\right)$ since, otherwise, $\sigma_{i}(N, v)=\sigma_{j}(N, v)=\sigma_{j}\left(N, v^{t}\right)>$ $\sigma_{i}\left(N, v^{t}\right)$, contradicting AM. Let $\hat{t} \in\left(0, t^{*}\right)$ be such that $2 \cdot\left(v^{t^{*}}(N)-v^{\hat{t}}(N)\right) \leq \sigma_{j}\left(N, v^{t}\right)-$ $\sigma_{i}\left(N, v^{t}\right)$. By $\mathrm{PO}$ and $\mathrm{AM}$, we obtain

$$
\begin{aligned}
2 \cdot\left(v^{t^{*}}(N)-v^{\hat{t}}(N)\right) \leq & \sigma_{j}\left(N, v^{t}\right)-\sigma_{i}\left(N, v^{t}\right) \\
\leq & \sigma_{j}\left(N, v^{t^{*}}\right)-\sigma_{i}\left(N, v^{t^{*}}\right) \\
= & \sigma_{j}\left(N, v^{\hat{t}}\right)-\sigma_{i}\left(N, v^{\hat{t}}\right)+\sigma_{j}\left(N, v^{t^{*}}\right)-\sigma_{j}\left(N, v^{\hat{t}}\right) \\
& -\left(\sigma_{i}\left(N, v^{t^{*}}\right)-\sigma_{i}\left(N, v^{\hat{t}}\right)\right) \\
\leq & \sigma_{j}\left(N, v^{\hat{t}}\right)-\sigma_{i}\left(N, v^{\hat{t}}\right)+\sum_{j \in N}\left(\sigma_{j}\left(N, v^{t^{*}}\right)-\sigma_{j}\left(N, v^{\hat{t}}\right)\right) \\
& -\left(\sigma_{i}\left(N, v^{t^{*}}\right)-\sigma_{i}\left(N, v^{\hat{t}}\right)\right) \\
\leq & \sigma_{j}\left(N, v^{\hat{t}}\right)-\sigma_{i}\left(N, v^{\hat{t}}\right)+v^{t^{*}}(N)-v^{\hat{t}}(N) .
\end{aligned}
$$

Hence, $v^{t^{*}}(N)-v^{\hat{t}}(N) \leq \sigma_{j}\left(N, v^{\hat{t}}\right)-\sigma_{i}\left(N, v^{\hat{t}}\right)$. Now, by $\mathrm{AM}$ and $\mathrm{BPF}, \sigma_{j}\left(N, v^{t^{*}}\right)=$ $\sigma_{j}\left(N, v^{\hat{t}}\right)$, contradicting $(15)$.

Now, we have all the tools to prove our characterization result.

Theorem 3. Let $\Gamma^{\prime} \subseteq \Gamma$ be closed under increments and $\sigma$ on $\Gamma^{\prime}$ be a Pareto optimal solution. Then, $\sigma$ on $\Gamma^{\prime}$ supports constrained welfare egalitarianism if and only if it satisfies $\mathrm{AM}$ and $\mathrm{BPF}$.

Proof. Let $\sigma$ be a Pareto optimal solution on $\Gamma^{\prime}$ supporting constrained welfare egalitarianism. From Equation (14) it follows AM. To check BPF, let $(N, v) \in \Gamma^{\prime}, t>0$, and $i, j \in N$ such that $\sigma_{i}(N, v)-\sigma_{j}(N, v) \geq t$. By $\mathbb{L} \mathbb{F}$ of $f^{C E}, f_{i}^{C E}(N, \sigma(N, v), t)=0$, and thus $\sigma_{i}\left(N, v^{t}\right)=\sigma_{i}(N, v)$ which proves $\mathrm{BPF}$.

To prove the reverse implication, let $\sigma$ be a Pareto optimal solution satisfying AM and BPF. Take $(N, v) \in \Gamma^{\prime}$ and $t>0$. Denote $\sigma(N, v)=x$ and $\sigma\left(N, v^{t}\right)=x^{t}$. Let $\mathcal{P}(x)=\left(N_{1}, N_{2}, \ldots, N_{k}\right)$ be the ordered partition of $N$ as defined in Section 2. We proceed by induction on $|\mathcal{P}(x)|$.

If $k=1$, by PO, $x_{i}=\frac{v(N)}{n}$ for all $i \in N$, where $|N|=n$. Hence, by Lemma $2, x_{i}^{t}=x_{j}^{t}$ for all $i, j \in N$, and by $\mathrm{PO}$, for all $i \in N$,

$$
x_{i}^{t}=\frac{v^{t}(N)}{n}=\frac{v(N)}{n}+\frac{t}{n}=x_{i}+f_{i}^{C E}(N, x, t),
$$


where the last equality comes from $\mathbb{E} \mathbb{T}$ of $f^{C E}$. Our induction hypothesis is that $x^{t}=$ $x+f^{C E}(N, x, t)$ whenever $k<\ell$ for some $\ell \in \mathbb{N}, \ell>1$. We now assume $k=\ell$. Take $i_{1} \in N_{1}$, with $n_{1}=\left|N_{1}\right|$ and $i_{2} \in N_{2}$. We distinguish two cases:

Case 1: $x_{i_{2}}-x_{i_{1}} \geq \frac{t}{n_{1}}$. By Lemma 2, for all $i, j \in N_{1}, x_{i}^{t}=x_{j}^{t}$, and AM together with BPF lead to $x_{i}^{t}=x_{i}$ for all $i \in N \backslash N_{1}$. Now, taking into account that $f^{C E}$ satisfies $\mathbb{L} \mathbb{F}$ and $\mathbb{E} \mathbb{T}$, we have that $x^{t}=x+f^{C E}(N, x, t)$.

Case 2: $x_{i_{2}}-x_{i_{1}}<\frac{t}{n_{1}}$. Let $t^{\prime}=n_{1}\left(x_{i_{2}}-x_{i_{1}}\right)$ and $\sigma\left(N, v^{t^{\prime}}\right)=x^{t^{\prime}}$. Note that $t-t^{\prime}>0$. By BPF, $x_{i}^{t^{\prime}}=x_{i}$ for all $i \in N \backslash N_{1}$. By Lemma 2 and PO, $x_{i}^{t^{\prime}}=x_{i}+\left(x_{i_{2}}-x_{i_{1}}\right)=x_{i_{2}}$ for all $i \in N_{1}$. Since $\left|\mathcal{P}\left(x^{t^{\prime}}\right)\right|=\ell-1$, by induction hypothesis $x^{t}=x^{t^{\prime}}+f^{C E}\left(N, x^{t^{\prime}}, t-t^{\prime}\right)$. Moreover, from $\mathbb{L} \mathbb{F}$ and $\mathbb{E} \mathbb{T}$ of $f^{C E}$ we receive $x^{t^{\prime}}=x+f^{C E}\left(N, x, t^{\prime}\right)$. Finally, from $\mathbb{P I}$ of $f^{C E}$ we obtain

$$
\begin{aligned}
x^{t} & =x^{t^{\prime}}+f^{C E}\left(N, x^{t^{\prime}}, t-t^{\prime}\right) \\
& =x+f^{C E}\left(N, x, t^{\prime}\right)+f^{C E}\left(N, x+f^{C E}\left(N, x, t^{\prime}\right), t-t^{\prime}\right) \\
& =x+f^{C E}(N, x, t) .
\end{aligned}
$$

Hence, $\sigma$ supports constrained welfare egalitarianism on any domain $\Gamma^{\prime}$ of games that is closed under increments.

Clearly, for one person games, PO implies both AM and BPF. However, for any domain of games $\Gamma^{\prime}$ that is closed under increments, and not contained in the class of one player games, by means of examples we show that each of the two properties in Theorem 3 is logically independent of the remaining property.

To this end choose an arbitrary game $\left(N_{*}, v_{*}\right) \in \Gamma^{\prime}$ with $\left|N_{*}\right| \geq 2$. Let $k \in N_{*}$ and $x \in \mathbb{R}^{N_{*}}$ given by

$$
x_{k}=\frac{v_{*}\left(N_{*}\right)}{\left|N_{*}\right|}-\left(\left|N_{*}\right|-1\right) \text { and } x_{i}=\frac{v_{*}\left(N_{*}\right)}{\left|N_{*}\right|}+1 \text { for all } i \in N_{*} \backslash\{k\} .
$$

Define $\sigma^{1}$ as follows. First, $\sigma^{1}\left(N_{*}, v_{*}\right)=x$. Now, for all $t \in \mathbb{R}$ such that $\left(N_{*}, v_{*}^{t}\right) \in \Gamma^{\prime}$, put $\sigma^{1}\left(N_{*}, v_{*}^{t}\right)=x+\frac{t}{\left|N_{*}\right|} \cdot e^{N}$, where, for any $\emptyset \neq S \subseteq N_{*}, e^{S} \in \mathbb{R}^{N_{*}}$ denotes the indicator function of $S$ defined by

$$
e_{i}^{S}=\left\{\begin{array}{l}
1, \text { if } i \in S, \\
0, \text { if } i \in N_{*} \backslash S
\end{array}\right.
$$

For all other $(N, v) \in \Gamma^{\prime}, \sigma^{1}(N, v)=\frac{v(N)}{|N|} e^{N}$. Then, $\sigma^{1}$ is a Pareto optimal solution that satisfies AM but violates BPF.

Moreover, for $(N, v) \in \Gamma^{\prime}$, define

$$
\sigma^{2}(N, v)= \begin{cases}x+t e^{\{k\}} & , \text { if }(N, v)=\left(N_{*}, v_{*}^{t}\right) \text { for some } t \leq 0 \\ \frac{v(N)}{|N|} e^{N} & , \text { for all other }(N, v) \in \Gamma^{\prime}\end{cases}
$$


Then $\sigma^{2}$ satisfies PO. Furthermore, $\sigma^{2}$ does not satisfy AM because for $\left|N_{*}\right|>t>0$ $\sigma_{i}^{2}\left(N_{*}, v_{*}^{t}\right)=\frac{v_{*}\left(N_{*}\right)+t}{\left|N_{*}\right|}<\frac{v_{*}\left(N_{*}\right)}{\left|N_{*}\right|}+1=\sigma_{i}^{2}\left(N_{*}, v_{*}\right)$ for all $i \in N_{*} \backslash\{k\}$. To show BPF, let $t>0,(N, v),\left(N, v^{t}\right) \in \Gamma^{\prime}$, and $i \in N$ such that $\sigma_{i}^{2}\left(N, v^{t}\right)>\sigma_{i}^{2}(N, v)$. It remains to show that $\sigma_{i}^{2}(N, v)-\sigma_{j}^{2}(N, v)<t$ for all $j \in N \backslash\{i\}$. A careful inspection of the definition of $\sigma^{2}$ shows that we may restrict the attention to the case that $N=N_{*}$ and $v=v_{*}^{t_{1}}$ for some $t_{1} \leq 0$. Therefore $v^{t}=v_{*}^{t_{2}}$ where $t_{2}=t+t_{1}$. We distinguish two cases:

Case 1: $t_{2} \leq 0$. Then $i=k$ and the proof is finished because $\sigma_{k}^{2}(N, v)=x_{k}+t_{1} \leq x_{k}<$ $x_{j}=\sigma_{j}^{2}(N, v)$ for all $j \in N \backslash\{k\}$.

Case 2: $t_{2}>0$. For $i=k$ we have $\sigma_{i}^{2}(N, v)=x_{k}+t_{1}<x_{j}=\sigma_{j}^{2}(N, v)$ for all $j \in N \backslash\{i\}$. If $i \neq k$, then $\sigma_{i}^{2}\left(N, v^{t}\right)>\sigma_{i}^{2}(N, v)=\frac{v_{*}\left(N_{*}\right)}{\left|N_{*}\right|}+1$ implies $\sigma^{2}\left(N, v^{t}\right)=\frac{v_{*}^{t_{2}}\left(N_{*}\right)}{\left|N_{*}\right|} e^{N_{*}}$, hence, $t_{2}>\left|N_{*}\right|$. However, $\sigma_{i}^{2}(N, v)-\sigma_{k}^{2}(N, v)=x_{i}-x_{k}-t_{1}=\left|N_{*}\right|-t_{1}<t_{2}-t_{1}=t$ so that the proof is also finished in this case.

To finish this section, we analyze constrained welfare egalitarianism for some specific domains of games.

A game $(N, v)$ is convex if and only if $v(S)+v(T) \leq v(S \cup T)+v(S \cap T)$, for every $S, T \subseteq N$. The set of convex games is denoted by $\Gamma_{v e x}$. A game $(N, v)$ is balanced if and only if it has a nonempty core (Bondareva, 1963; Shapley 1967). Let $(N, v)$ be an arbitrary game. An upper vector (Bennett, 1983) is a vector $x \in \mathbb{R}^{N}$ such that $x(S) \geq v(S)$ for all $S \subseteq N$. By $U P(N, v)$ we denote the set of upper vectors. The core is large (Sharkey, 1982) if for all $y \in U P(N, v)$, there exists $x \in C(N, v)$ such that $x \leq y$. By $\Gamma_{b a l}$ and $\Gamma_{l c}$ we denote the set of balanced games and games with large core, respectively. The domains of convex games, balanced games, and games with large core are closed under increments. ${ }^{8}$ On convex games, the egalitarian solution of Dutta and Ray (1989), denoted by $L$, selects the unique core element that Lorenz dominates every other core point. ${ }^{9}$ That is, given $(N, v) \in \Gamma_{\text {vex }}$,

$$
L(N, v) \in C(N, v) \text { and } L(N, v) \succ_{\mathcal{L}} y \text { for all } y \in C(N, v) \backslash\{L(N, v)\} .
$$

Given $(N, v) \in \Gamma_{b a l}$, the lexmax solution (Arin et al., 2003) is defined as

$$
\operatorname{Lmax}(N, v)=\left\{x \in C(N, v) \mid \hat{x} \preceq_{\text {lex }} \hat{y} \text { for all } y \in C(N, v)\right\},
$$

where $\hat{x}, \hat{y} \in \mathbb{R}^{N}$ denote the vectors obtained from $x \in \mathbb{R}^{N}$ and $y \in \mathbb{R}^{N}$, respectively, by ordering its coordinates in a non-increasing way. Recall that for any two vectors $x, y \in$ $\mathbb{R}^{N}$, we say that $x \preceq_{\text {lex }} y$ if $x=y$ or there exists $k \in\{1, \ldots,|N|\}$ such that $x_{i}=y_{i}$ for $1 \leq$ $i \leq k-1$ and $x_{k}<y_{k}$. It is known that the lexmax solution is a singleton and, moreover,

\footnotetext{
${ }^{8}$ For a discussion of prosperity properties see van Gellekom et al. (1999).

${ }^{9}$ This is not the original definition, but is shown in Dutta and Ray (1989) to coincide with their egalitarian solution for convex games.
} 
it is Lorenz undominated within the core. To harmonize egalitarian considerations and particular interests, Arin et al. (2003) introduce the following definition.

Definition 3. A solution $\sigma$ on a domain $\Gamma^{\prime} \subseteq \Gamma_{\text {bal }}$ is called egalitarian if for all $(N, v) \in$ $\Gamma^{\prime}, \sigma(N, v) \in C(N, v)$ and there is no $y \in C(N, v)$ such that $y \succ_{\mathcal{L}} \sigma(N, v)$.

As the following example shows, on the domain of balanced games there is no egalitarian solutions supporting constrained welfare egalitarianism.

Example 2. Assume $|U| \geq 3$. Consider the game $(N, v)$ where $N=\{1,2,3\}, v(\{i\})=$ $v(\{2,3\})=0$, for all $i \in N$, and $v(\{1,2\})=v(\{1,3\})=v(\{1,2,3\})=1$. Let $\sigma$ be an egalitarian solution on $\Gamma_{b a l}$. Then, $C(N, v)=\{(1,0,0)\}$ and $\left(\frac{2}{3}, \frac{2}{3}, \frac{2}{3}\right) \in C\left(N, v^{1}\right)$, which leads to $\sigma(N, v)=(1,0,0)$ and $\sigma\left(N, v^{1}\right)=\left(\frac{2}{3}, \frac{2}{3}, \frac{2}{3}\right)$. Note that $\sigma$ violates AM and, consequently, it does not support constrained welfare egalitarianism on $\Gamma_{b a l}$.

Next, we prove that any egalitarian solution that satisfies AM also satisfies constrained welfare egalitarianism.

Theorem 4. Let $\sigma$ be an egalitarian solution on $\Gamma^{\prime} \subseteq \Gamma_{\text {bal }}$ closed under increments satisfying AM. Then, $\sigma$ supports constrained welfare egalitarianism on $\Gamma^{\prime}$.

Proof. Suppose there is $(N, v) \in \Gamma^{\prime}$ and $t>0$ such that $\sigma\left(N, v^{t}\right) \neq x^{*}+f^{C E}\left(N, x^{*}, t\right)$, where $x^{*}=\sigma(N, v)$. By AM, there exists $z \in \mathbb{R}_{+}^{N}$ with $z(N)=t$ such that $\sigma\left(N, v^{t}\right)=$ $x^{*}+z$. By Lemma $1, x^{*}+f^{C E}\left(N, x^{*}, t\right) \succ_{\mathcal{L}} x^{*}+z$. But $x^{*}+f^{C E}\left(N, x^{*}, t\right) \in C\left(N, v^{t}\right)$, which leads to a contradiction.

Since both the Dutta and Ray (1989) solution and the lexmax solution are egalitarian solutions satisfying AM in $\Gamma_{v e x}$ and $\Gamma_{l c}$, respectively (see, for instance, Hokari and van Gellekom, 2002; Arin et al., 2003), from Theorem 4 we receive the following corollary.

Corollary 3. (i) On $\Gamma_{v e x}$, the egalitarian solution of Dutta and Ray (1989) supports constrained welfare egalitarianism.

(ii) On $\Gamma_{l c}$, the lexmax solution supports constrained welfare egalitarianism.

\section{References}

[1] Arin, J., J. Kuipers, and D. Vermeulen (2003) Some characterizations of egalitarian solutions on classes of TU-games. Mathematical Social Sciences, 46: 327-345.

[2] Bennett, E. (1983) The aspiration approach to predicting coalition formation and payoff distribution in sidepayment games. International Journal of Game Theory, 12: $1-28$. 
[3] Bondareva, O. N. (1963) Some applications of linear programming methods to the theory of cooperative games. Problemi Kibernitiki, 10: 119-139.

[4] Chun, Y. (1989) A noncooperative justification for egalitarian surplus sharing. Mathematical Social Sciences, 17: 245-261.

[5] Dutta, B., and D. Ray (1989) A concept of egalitarianism under participation constraints. Econometrica, 57: 615-635.

[6] Hokari, T. and van Gellekom (2002) Population monotonicity and consistency in convex games: Some logical relations. International Journal of Game Theory, 31: 593-607.

[7] Ju, B-G., and J. Moreno-Ternero (2018) Entitlement theory of justice and end-state fairness in the allocation of goods. Economics and Philosophy, 34: 317-341.

[8] Megiddo, N. (1974) On the monotonicity of the bargaining set, the kernel, and the nucleolus of a game. SIAM Journal on Applied Mathematics, 27: 355-358.

[9] Moreno-Ternero, J., and J. E. Roemer (2012) A common ground for resource and welfare egalitarianism. Games and Economic Behavior, 75: 832-841.

[10] Moulin, H. (1987) Equal or proportional division of a surplus, and other methods. International Journal of Game Theory, 16: 161-186.

[11] Pfingsten, A. (1991) Surplus-sharing methods. Mathematical Social Sciences, 21: 287-301.

[12] Pfingsten, A. (1998) Cheating by groups and cheating over time in surplus sharing problems. Mathematical Social Sciences, 36: 243-249.

[13] Sharkey, W. (1982) Cooperative games with large cores. International Journal of Game Theory, 11: 175-182.

[14] Shapley, L.S. (1967) On balanced sets and cores. Naval Research Logistics Quarterly, 14: $453-460$.

[15] Shapley, L.S. (1971) Cores of convex games. International Journal of Game Theory, 1: $11-26$.

[16] Timoner, P., and J. M. Izquierdo (2016) Rationing problems with ex-ante conditions. Mathematical Social Sciences, 79: 46-52.

[17] van Gellekom, J., Potters, J., and J. Reijnierse (1999) Prosperity properties of TUgames. International Journal of Game Theory, 28: 211-227.

[18] Young, H.P. (1988) Distributive justice in taxation. Journal of Economic Theory, 44: 321-335. 